\title{
The classification of intestinal polyposis
}

To the Editor:

A recent publication ${ }^{1}$ in Nature Genetics quoted our paper ${ }^{2}$ on hereditary mixed polyposis syndrome (HMPS) and alluded to the Singapore Chinese families with HMPS as having juvenile polyposis syndrome (JPS). Diagnosis with JPS requires the presence of numerous (up to 50) juvenile polyps in the gastrointestinal tract and is often made in children before adolescence ${ }^{3}$. We wish to clarify that our diagnostic criteria for HMPS, "presence of mixed (e.g., mixed 'hyperplastic-adenomatous', mixed 'juvenile-hyperplastic' or 'serrated adenomas') polyp(s) in the colon or rectum and the absence of upper gastrointestinal abnormalities as documented by certified pathologists"2, is inclusive and similar to the definition used in the first description of HMPS in a large Ashkenazi family ${ }^{4}$. On the basis of these criteria, we have thus far identified nine families with HMPS in the Singapore Polyposis Registry, five of which have germline mutations in the BMPR1A gene (encoding bone morphogenetic protein receptor 1A). Four different mutations were identified, as two families shared the same ancestral mutation. Detailed morphology of these 'mixed' polyps was presented in Figure 1 of an earlier report ${ }^{5}$.

We wish to emphasize that none of the Singapore families with HMPS were diagnosed as having JPS. The most defining characteristic of these families with HMPS was the presence of mixed hyperplastic-adenomatous polyps among the adenomatous (up to 100) polyps throughout the colon and rectum. Juvenile polyps were not documented in all affected family members, and, when reported, they were found to be frequently associated with hyperplastic components. Detailed histology of the polyps from one such affected individual over a timeline of 11 years (Table 1 of ref. 2) showed distinct morphological differences in HMPS and JPS. In fact, the difficulty in diagnosis rests in distinguishing HMPS from attenuated familial adenomatous polyposis (FAP) rather than from JPS. FAP is another syndrome inherited in an autosomal dominant manner that is characterized by up to thousands of adenomatous polyps in the colon and rectum, and, if left untreated, it eventually leads to the development of colorectal cancers. The majority of FAP cases are caused by inactivation of the APC gene (encoding adenomatous polyposis coli) ${ }^{6}$.

Moreover, the mean age at diagnosis of the Singapore individuals with HMPS was 43 (range of 16-61) years, which is significantly older than for JPS, which is typically diagnosed before puberty.

The report by Jaeger et al. identified the founder mutation in Ashkenazi families with HMPS ${ }^{1}$. The 40-kb duplication upstream of GREM1 leads to increased ectopic expression of GREM1, a BMP antagonist. This finding suggests that genetic heterogeneity exists in HMPS but does not negate our finding that, for the majority of Chinese individuals with HMPS, the disease-causing mutations are in $B M P R 1 A$. Recognizing that mutations in $B M P R 1 A$ rather than $A P C$ are the likely cause of the mixed morphology polyps has enabled us to rapidly screen for the germline defect and offer presymptomatic testing to members of the families with HMPS. Recently, BMPR1A mutations were reported in an Irish family with HMPS, indicating that inactivating BMPR1A can cause HMPS across different populations ${ }^{7}$.

\section{AUTHOR CONTRIBUTIONS}

P.Y.C. supervised experiments, analyzed data and wrote the manuscript. K.H.L. provided pathological input. P.K.K. and C.L.T. provided clinical input and obtained samples.

\section{COMPETING FINANCIAL INTERESTS}

The authors declare no competing financial interests.

Peh Yean Cheah ${ }^{1-3}$, Kiat Hon Lim ${ }^{4}$, Poh Koon Koh ${ }^{1}$ \& Choong Leong Tang ${ }^{1}$

${ }^{1}$ Department of Colorectal Surgery, Singapore General Hospital, Singapore. ${ }^{2}$ Saw Swee Hock School of Public Health, National University of Singapore (NUS), Singapore. ${ }^{3}$ Duke-NUS Graduate Medical School, National University of Singapore, Singapore. ${ }^{4}$ Department of Pathology, Singapore General Hospital, Singapore.

e-mail: cheah.peh.yean@sgh.com.sg

1. Jaeger, E. et al. Nat. Genet. 44, 699-705 (2012).

2. Cheah, P.Y. et al. Am. J. Gastroenterol. 104, 30273033 (2009).

3. Merg, A. \& Howe, J.R. Am. J. Med. Genet. 129C 44-55 (2004).

4. Whitelaw, S.C. et al. Gastroenterology 112, 327-334 (1997).

5. Cao, X. et al. J. Med. Genet. 43, e13 (2006).

6. Cheah, P.Y. Crit. Rev. Oncol. Hematol. 69, 45-55 (2009).

7. O'Riordan, J.M. et al. Colorectal Dis. 12, 570-573 (2012).

Tomlinson et al. reply:

In response to our report that germline GREM1 mutations cause hereditary mixed polyposis syndrome (HMPS), Cheah and colleagues write that some families under their care have HMPS caused by germline BMPR1A mutations ${ }^{1,2}$. The original family with HMPS was SM96, described over 50 years ago at St Mark's Hospital, London, by H.J.R. Bussey. The name HMPS was used because the family had polyps of unusual and highly variable histology ${ }^{3}$, to an extent that had not previously been reported and has not been found since in families without GREM1 mutations ${ }^{4}$.

The affected individuals reported by Cheah et al. do have some phenotypic similarities to the prototypical SM96 family and to other families with GREM1 mutations ${ }^{1,2,4}$. This is not unexpected because GREM1 and BMPR1A both function in the bone morphogenetic protein (BMP) signaling pathway. However, germline $B M P R 1 A$ mutations are a wellestablished cause of juvenile polyposis syndrome (JPS $)^{5}$, and, in the BMPR1Amutant families that are classified by Cheah et al. as having HMPS, the almost pathognomonic juvenile polyps are often present, even if the presentation is atypical for JPS. In fact, we have reported that BMPR1A-mutant individuals with JPS can develop adenomas and mixed polyps in addition to juvenile polyps ${ }^{6}$. 